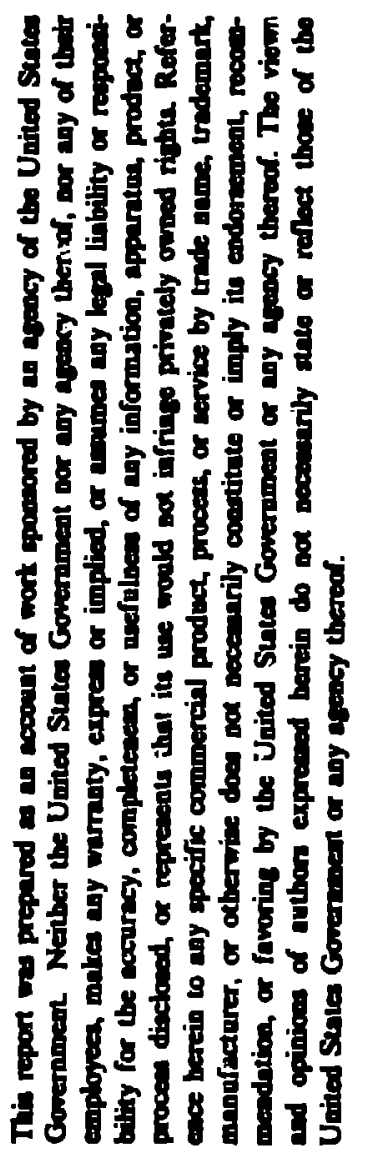

Preformed Transient Gas Channels for Laser Wakefield Particle Acceleration

Author(s):

Willian M. Wood

OSA Annual Meet1ng ' 94

October 2-7, 1994

Dallas, Texas

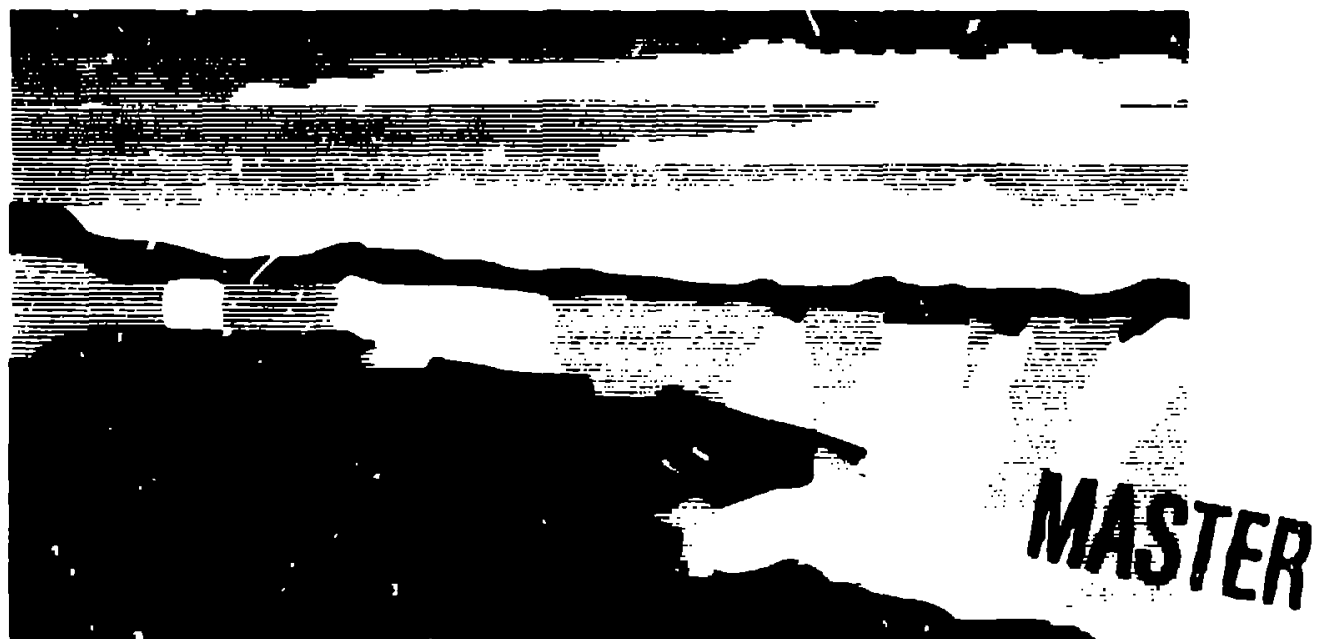




\section{Preformed Transient Gas Channels for Laser Wakefield Particle Acceleration}

Acceleration of electrons by laser-driven plasma wake fields is limited by the range over which a laser pulse can maintain its intensity. This distance is typically given by the Rayleigh range for the focused laser beam, usually on the order of $0.1 \mathrm{~mm}$ to $1 \mathrm{~mm}$. For practical particle acieleration, interaction distances on the order of centimeters are required. Therefore, some means of guiding high intensity laser pulses is necessary.

Light intensities on the order of a few times $10^{17} \mathrm{~W} / \mathrm{cm}^{2}$ are required for laser wakefield acceleration schemes using near $\mathbb{I R}(800 \mathrm{~nm}$ to 1 micron) radiation. Gas densities on the order of or greater than $10^{17} \mathrm{~cm}^{-3}$ are also needed. Laser-atom interaction studies in this density and intensity règime $\left(I_{\text {peak }}>10^{14} \mathrm{~W} / \mathrm{cm}^{2}, 10^{17} \mathrm{~cm}^{-3}<\right.$ $\rho \lesssim 10^{21} \mathrm{cr}^{1-3}$ ) are generally limited by the concomitant problems in beam propagation introduced by the creation of a plasma. ${ }^{1}$ In addition to the interaction distance limit imposed by the Rayleigh range, defocusing of the high intensity laser pulse further limits the peak intensity which can be achieved. Because of the defocusing and beam break-up, these types of experiments often have a large uncertainty about the actual plasma density and laser intensity conditions which occur in the interaction region.

To solve the problem of beam propagation limitations in laser-plasma wakefield experiments, two potential methods for creating transient propagation channels in gaseous targets are investigated. Each method comprises a two-pulse experiment. The first involves creation of a charge-neutral channel in a gas by an initial laser pulse, which then is ionized by a second, ultrashort, high-intensity pulse to creste a waveguide. The second method involves the ionization of a gas column by an ultrashort pulse; a transient waveguide is formed by the subsequent expansion of the heated plasma into the neutral gas.

\section{Propagation of Light in Laser-produced Plasmas}

The condition for channeling of a laser pulse is that the refraction of the beam due to variations in the index balances the diffraction of the beam. In an axially symmetric geometry the index of refraction can be written, to first order, with a quadratic spatial dependence on $r$ :

$$
n(r)=n_{0}\left(1-\alpha r^{2}\right)
$$

The constant $\alpha$ is positive for focusing, negative for defocusing. To balance diffraction, the minimum value that $\alpha$ can have is given by the expression:

$$
\alpha=\frac{\lambda^{2}}{8 \pi^{2} \mathrm{r}_{\mathrm{o}}^{4}}
$$

Here, $\lambda$ is the vicuum wavelength of the laser, and $r_{0}$ is the intensity 1/e radius of the laser beam. If $\alpha$ is greater than the value in equation (2), the beam will converge.

When a gas is ionized by a laser pulse, the higher intensity on axis typically yields a plasma density which is greater on axis. This results in an index profile with a negative $\alpha$, causing a defocusing of the lase: beam, which limits the peak intensity. It is possible to create a density profile in the ambient gas that offsets the increased ionization on axis with a decreased number density on axis. To illustrate this, the condition for focusing or defocusing can be translated to a condition on the plasma density profile. To quadratic 
order in a Taylor's series, the index of refraction can be written in terms of the plasma density, $\mathbf{N}$ :

$$
\mathrm{n} \approx 1-\frac{\mathrm{N}(\mathrm{r}) \mathrm{e}^{2}}{2 \epsilon_{\mathrm{o}} \mathrm{m}_{\mathrm{e}} \omega_{\mathrm{p}}^{2}}(\mathrm{MKS})
$$

For a quadratic density profile, where the plasma density aifference at $r_{0}$ is $\left(N_{0}+\Delta N\right)$, the index becomes

$$
\mathrm{n} \approx \mathrm{n}_{\mathrm{o}}-\frac{\mathrm{e}^{2}}{2 \epsilon_{\mathrm{o}} \mathrm{m}_{\mathrm{e}} \omega_{\mathrm{p}}{ }^{2}} \frac{\Delta \mathrm{N} \mathrm{r^{2 }}}{\mathrm{r}_{\mathrm{o}}^{2}}(\mathrm{MKS})
$$

Comparing this expression with the expression for $\alpha$ in equation (2) above gives the condition on the plasma density difference between the center and at radius $r_{0}$, the laser 1/e spot size:

$$
\Delta N=\frac{\epsilon_{0} m_{e} c^{2}}{e^{2} r_{0}^{2}}
$$

This says that in order for the refraction to balance the diffraction of a laser beam with intensity $1 / e$ radius $r_{n}$, the plasma density at a distance $r_{0}$ from the center must be greater than the ،'ensity on axis hy this number $\Delta \mathrm{N}$. For a laser spot size of one micron radius, this number is $2.8 \times 10^{19} \mathrm{~cm}^{-3}$. Note that this condition for channeling is independent of wavelength, and depends only on the laser spot size.

\section{Heating of a Gas Column}

When a column of gas is heated with axial symmetry, the gas expands to leave a density "hole" along the axis. The depth of this "hole," the time in which it develops, and the transverse dimensions all depend upon the spatial profile of the initial heating, the time over which the heat is deposited, and the gas into which the heat is deposited. Two methods are described for heating such a column of gas. The first takes advantage of the process of photodissociation to transfer laser energy io thermal motion, leaving a chargeneutral density channel. The second uses inverse bremmstrahlung after multiphoton ionization to thermally excite the gas mulecules. Evolution of the gas density, $\rho(r)$, is calculated numerically using the fluid flow equations ${ }^{2}$. The plasma density profile is figured as the locally averaged degree of ionization of the individual gas molecules multiplied by the number density of the gas molecules:

$$
N_{e}(r)=D_{i}(r) \rho(r)
$$

The degree of ionization for ultrafast laser illumination, $D_{i}(r)$, can be estimated using ADK thecry ${ }^{3}$. Below, results of modeling both the heated expansion of a gas and the ultrafast ionization are presented.

\section{Preformed Channel Using Photodissociation}

Many molecules have an abscrption in the near UV which corresponds to a photodissociation energy. The fourth harmonic of a $\mathrm{Nd}$ :YAG laser is an excellent source to initiate this type of process. Other wavelengths can, of course, be used. The resulting products of the photodissociation have a strong thermal motion. and the spatial temperature profile follows that of the laser intensity. Table I lists several gases with the 
absorption cross section for $266 \mathrm{~nm}$, along with the resulting energy per dissociated particle."

\begin{tabular}{|c|c|c|}
\hline Gas & $\sigma_{\mathrm{abs}}\left(10^{-20} \mathrm{~cm}^{2}\right)$ & Temp. (eV) \\
\hline \hline $\mathrm{Cl}_{2}$ & 1.5 & 1.0 \\
\hline $\mathrm{F}_{2}$ & 1.9 & 1.5 \\
\hline Acetone & 4.5 & 1.0 \\
\hline $\mathrm{H}_{2} \mathrm{O}_{2}$ & 11.5 & 1.2 \\
\hline $\mathrm{HI}$ & 15.3 & 0.8 \\
\hline $\mathrm{SO}_{2}$ & 42 & 1.5 \\
\hline
\end{tabular}

Table 1: Photodissociation absorption cross section at $266 \mathrm{~nm}$ and energy per dissociated molecule for severa! gases.

The modeling presented here uses a 10 ns Gaussian temporal pulse. Figure 1 shows the time evolution of the density as a function of radius in steps of $0.5 \mathrm{~ns}$, starting at a time $-4.5 \mathrm{~ns}$ before the peak of the $10 \mathrm{~ns}$ heating pulse. A peale temperature of $1 \mathrm{eV}$ is

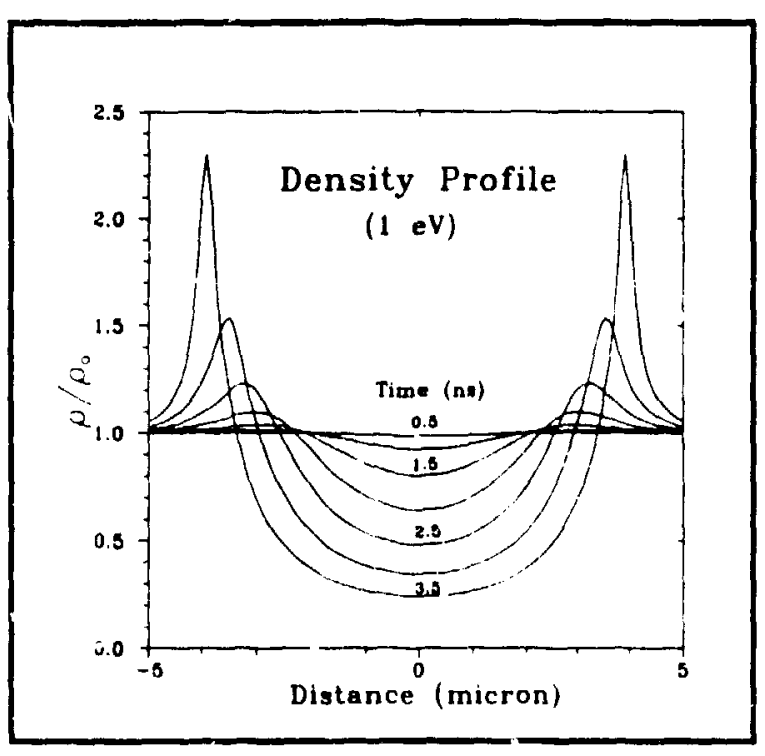

Figure 1: Time evolution of the density profile as a funcion of radial position for a gas heated to $1 \mathrm{eV}$ on axis. Initial heating pulse profile has 2 micron radius. created on axis. a typical temperature after photodissociation given in table 1.

In figure 2, calculations of ultrafast ionization of a thin slab of 'as are compared for the cases of a): uniform ambient gas density and b): a preformed, neutral density channe, corresponding to the curve at $3.5 \mathrm{~ns}$ in figure $\therefore$. The ultrefast pulse is 100 fs FWHM with a peak intensity of $10^{17} \mathrm{~W} / \mathrm{cm}^{2}$ and an intensity $1 / \mathrm{e}$ radius of $2.6 \mu \mathrm{m}$. This spot sizc is chosen to assure that more than $99 \%$ of the ionizing pulse energy is within the "walls" of the channel. For case a) using a uniform gas density, it is seen that at approximately -140 fs (before the peak of the pulse) an index profile which is "concave up," or defocusing, has formed. Furthermore, it is observed that all subsequent index profiles are defocusing. In contrast, case b) with an initial preformed neutral channel results in an index profile which is only slightly defocusing at -140 fs, but is "concave down" or focusing for all subsequent times. The actual "depth" of the resulting channel can be adjusted by changing the ambient gas density. As the ultrashort ionizing pulse proceeds through the preformed channel, it essentially writes its own waveguide. The necessary difference in the index between the axis and a position of 2.6 microns can be calculated using the condition given in equation (5) above. With a wavelength of 800 
$\mathrm{nm}$, the calcuiaied index of refraction difference required is $5 \times 10^{-3}$. The corresponding plasma density difference to channel the pulse would require an initial ambient gas

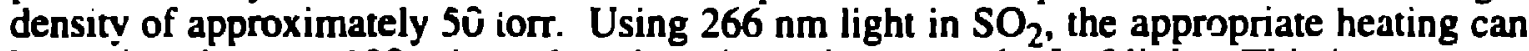
be arhieved over a 100 micron length region using several $\mu \mathrm{J}$ of light. This increases to several $\mathrm{mJ}$ when centimeter-long regions are needed. For larger wave guides, smaller

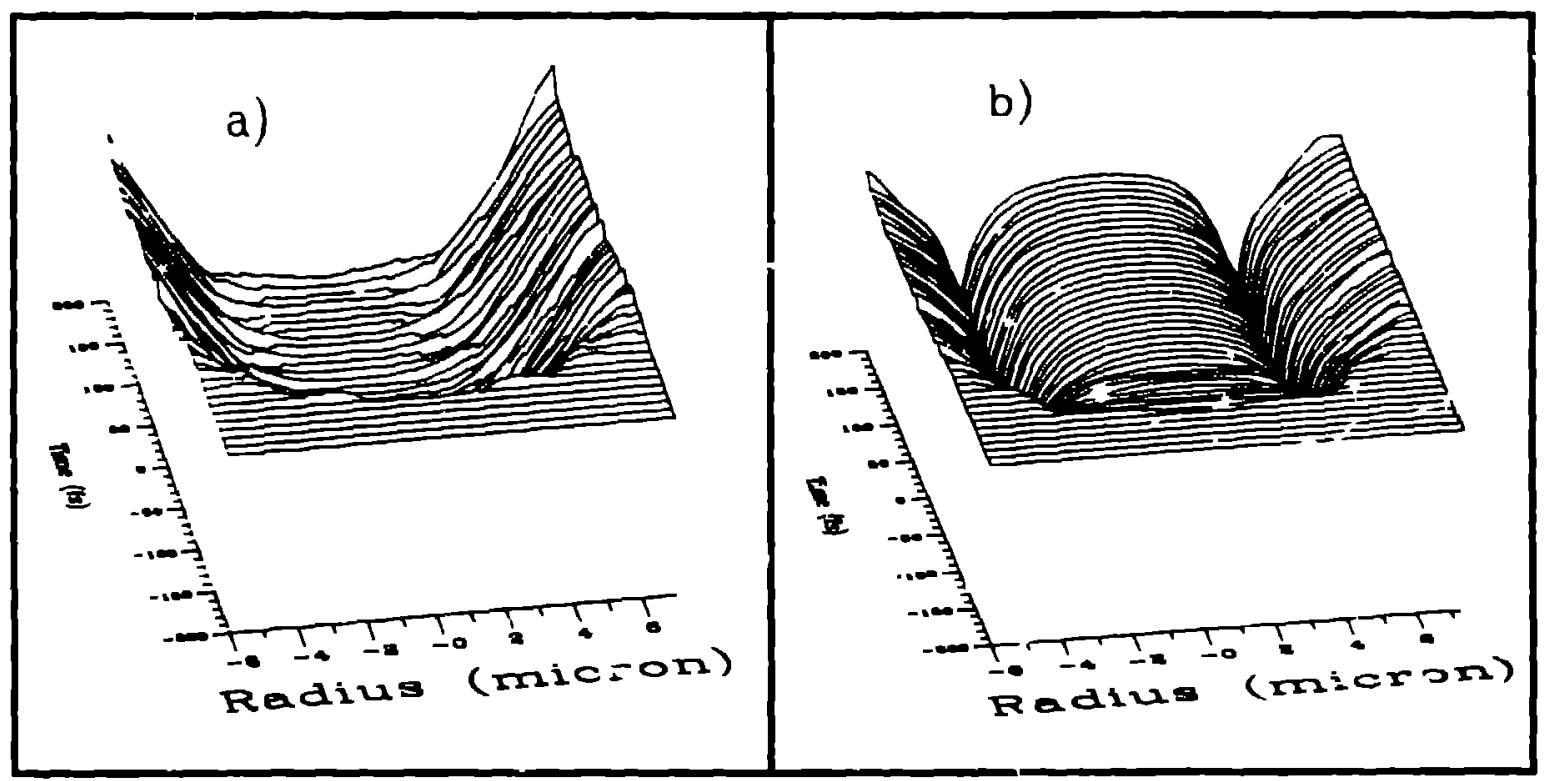

Higure 2: Radial dependence of the index of refraction as a function of time as the result of ultrafast ionization. a) Index of a thin slab of gas with initial uniform density. b) Index of a thin slab of gas with initial density given by the curve at $3.5 \mathrm{~ns}$ in figure 1 . The spot size of the ionizing ultrafast pulse is $2.6 \mu \mathrm{m} 1 / \mathrm{e}$ intensity radius.

pressures can be used.

\section{Plasma Channel Using Ultrafast Ionization and Heating}

When a gas is ionized by an intense, ultrashort pulse. calculations indicate that the final temperature immediately following the ionizing pulse is on the order of tens of eV. 5 Figure 3 shows plasma densities calculated using an initial temperature profile with peak on-axis of $20 \mathrm{eV}$. Note that although the density expansion is qualitatively the same as in the case of photodissociation, the time scale is approximately one tenth of that shown figure 1. Calculations for the necessary ambient density are essentially the same as those above for the photolissociation case.

Two problems exist with this method of channel creation. First, defocusing of the ionizing pulse as the plasma is created makes it difficult to achieve the assumed temperature profile using standard focusing optics. This can be overcome by using an axicon lens geomatry for creating the

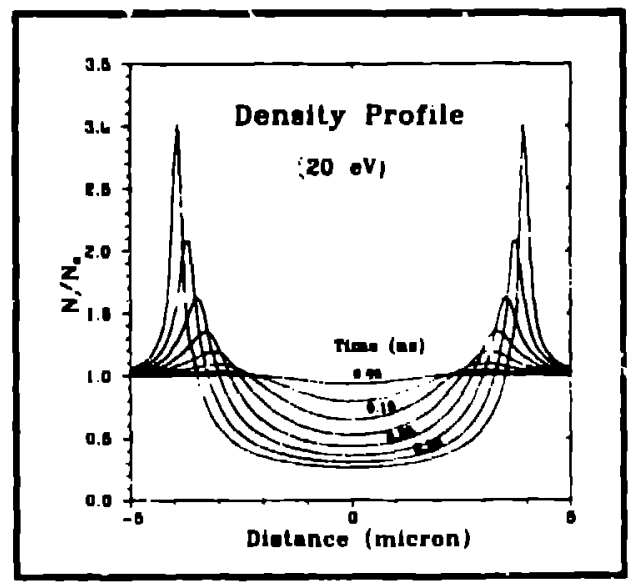

Figure 3: Plasma density as a function of radial position at different times following ionization by an ultrashort pulse. channel. Second, a great deal of energy is required to form channels of extended length using ultrashort pulses: over $50 \mathrm{~mJ}$ in an ultrashort pulse, iompared to the several $\mathrm{mJ}$ required using photod:ssociation. 


\section{Use of an Axicon Lens}

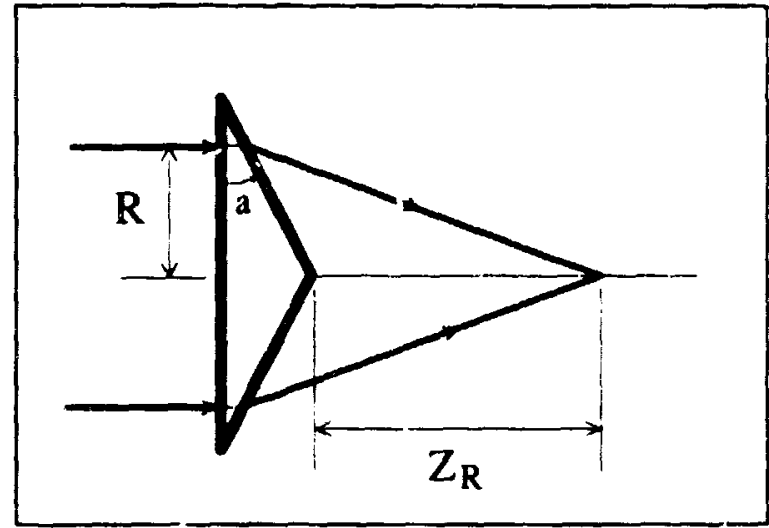

Figure 4: Focusing of an axicon lens. The incident beam radius is given by $R$, and the length of the focused region is given by $Z_{R}$. The angle of the axicon is indirated by "a."
With an axicon lens, a line focus of arbitrary length can be achieved. ${ }^{6}$ In the case of ultrashort pulse ionization, there is no problem with defocusing on axis. However, significantly more energy is needed to ionize the gas with an ultrashort pulse in this geometry. Calculations indicate on the order of $50 \mathrm{~mJ}$ to create a region $1 \mathrm{~cm}$ long. For both heating schemes, the line focus provides a means of extending the channel to hundreds of times tne confocal length associated with standard focusing optics. For the modeling presented above, the initial temperature spatial profile must be modified from a Gaussian to a Bessel function shape; however, qualitative aspects of the analysis remain the same.

With the axicon, the important parameters of the focal region are given by:

$$
\begin{aligned}
& \mathrm{Z}_{\mathrm{R}} \approx \frac{\mathrm{R}}{\mathrm{na}} \\
& \mathrm{r}_{\mathrm{f}} \approx \frac{\lambda}{\mathrm{a}}
\end{aligned}
$$

Here, $R$ is the radius of the incident laser spot, $Z_{R}$ is the length of the focal region, $r_{f}$ is the focal spot size, and " $\mathrm{a}$ " is the angle of the axicon lens.

\section{Wakefield Acceleration}

It is now possible to alculate potential acceleration capabilities using a channel as described above. ${ }^{7}$ For the calculated pressure of 50 torr, the appropriate pulse duration to excite the maximum acceierating field is approximately 70 fs. A light pulse from a Ti:Sapphire system is assumed, with wavelength of $800 \mathrm{~nm}$. The maximum accelerating field depends linearly upon the peak intensity. Table 2 gives the accelerating field for several pulse energies focused to $1 \mathrm{~cm}$ length using an axicon focusing elemert, and the resulting potential acceicration of electrons througt: that length. Focused spot size is assumed to be approximately $3 \lambda$.

\begin{tabular}{|c|c|c|c|}
\hline Pulse Energy $(\mathrm{mJ})$ & Peak I $\left(\mathrm{i} 0^{17} \mathrm{~W} / \mathrm{cm}^{2}\right)$ & Accel. Field $(\mathrm{GeV} / \mathrm{m})$ & Max. Accel. $(\mathrm{MeV})$ \\
\hline 0.13 & 0.1 & 0.16 & 1.6 \\
\hline 1.3 & 1.0 & 1.6 & 16 \\
\hline 13 & 10 & 16 & 160 \\
\hline
\end{tabular}

In summary, two methods for heating an axially symmetric region of gas are described. The resulting gas expansion a transient channel through which ultrashort. high-intensity laser pulses can be guided. In the case of photodissociative heating, 
significantly less energy is required to create extended chiannels. Channels can be created in gas pressures above approximately 1 torr, depending on the size of the channel desired. For a gas density of 50 torr. a 70 fs puise with peak inteilsity of $10^{16} \mathrm{~W} / \mathrm{cm}^{2}$ can provide an accelerating field of approximateiy $16 \mathrm{GeV} / \mathrm{m}$. In conjunction with the extencied interation region provided by the axicon lens, this con result in acceleration energies in the hundreds of $\mathrm{MeV}$.

\section{References}

1. P. Monot et.al., "Focusing limits of a terawatt laser in an underdense plasma," Jour. Opt. Soc. Am. b 9, 1579. (1992)

2. Zel'dovich, Y.B. and Y.P Raizer, Physics of Shock Waves and High-Temperature Hydrodynamic Phenomena, Academic Press, N.Y., 1966.

3. M.V. Ammosov et.al., "Tunnei ionization of cornplex atoms and of atomic ions in an alternating electromagnetic field," Sov. Phys. JETP 64, 1191. (1986)

4. Finlayson-Pitts, B.J. and J.N. Pitts, Jr., Atmospheric Chemistry: Fundarnentals and Experimental Techniques, Wiley \& Sons, N.Y. 1986.

CRC Handbook, 75th Editior, 1994. pps c. ' through 9-73.

5. Penetrante, B.M. and J.N. Bardsley, Phys. Rev. A 4.3, 3100. (1991)

6. Korobkin, V.V. et.al., Sov. J. Quant. Elec. 16, 178. (1986)

7. T. Tajinıa and J.M. Dawson, Phys. Rev. Lett. 43, $267 . \quad$ (1979) P. Sprangle et. al., Appl. Phys. Lett. 53, 2146. (1938) 